\title{
Moralische Probleme und ethische Fragen von Lehrenden der Erwachsenen- und Weiterbildung - Ein Modell für die Forschung zur erwachsenenpädagogischen Bereichsethik
}

\author{
Nils Bernhardsson-Laros iD
}

Eingegangen: 12. Dezember 2019 / Angenommen: 15. Februar 2020 / Online publiziert: 5. März 2020 (C) Der/die Autor(en) 2020

Zusammenfassung Die Kompetenz, ethisch-moralisch zu handeln, wird im Rahmen von Kompetenzkatalogen für Lehrkräfte der Erwachsenen- und Weiterbildung als wichtiges Lernfeld ausgewiesen. Allerdings fehlt bisher Forschung, welche die Perspektive von Lehrenden auf eine Berufsethik für die Erwachsenen- und Weiterbildung genauer beleuchtet. Gegenstand des Beitrags ist eine qualitative Interviewstudie, welche Fragen nach ethisch relevanten Handlungssituationen, konkreten ethischen Fragen, pädagogisch-ethischen Überzeugungen und ethischen Orientierungen von Lehrkräften der Erwachsenen- und Weitbildung nachgeht. Um das Forschungsfeld zu erschließen, wird auf Basis der Studienergebnisse ein vierdimensionales Modell moralischer Probleme entwickelt.

Schlüsselwörter Erwachsenenbildung · Weiterbildung · Bereichsethik · Berufsethik · Ethik · Moral · Professionalisierung · empirische Forschung

\section{Moral problems and ethical questions of adult educators-A model for research on applied ethics within the field of adult education}

Abstract The ability to act according to an ethical and moral perspective is identified as an important area of learning within competence frameworks for teachers in adult education. However, so far there is a lack of research that sheds light on teachers' perspectives on professional ethics for adult educators. The article reports on a qualitative interview study, which investigates questions of ethically relevant action situations, specific ethical questions, pedagogical-ethical beliefs and ethical orientations of adult educators. Based on the findings, a four-dimensional model of

Dr. N. Bernhardsson-Laros $(\bowtie)$

Pädagogische Hochschule Freiburg, 79117 Freiburg, Deutschland

E-Mail: nils.bernhardsson-laros@ph-freiburg.de 
moral problems is being developed in order to provide a basis for further research on the topic.

Keywords Adult education - continuing education · professional ethics · ethics · moral $\cdot$ professionalization $\cdot$ empirical research

\section{Einleitung}

Auch wenn wir spätestens seit Luhmanns (1993) Warnung vor Moral wissen, dass moralische Kommunikation mit Risiken behaftet ist, scheint es unausweichlich zu sein, Erwachsenen- und Weiterbildung als moralische Praxis zu verstehen (Fuhr 2011; Schrader 2018), die, begleitet durch Forschung, diskursiv weiterentwickelt werden kann. Das GRETA-Kompetenzmodell, welches künftig als Grundlage für ein trägerübergreifendes Anerkennungsverfahren für Kompetenzen Lehrender in der Erwachsenen- und Weiterbildung dienen soll, weist die Ethik - hier unter der Bezeichnung ,professionelle Werthaltungen und Überzeugungen“ - neben dem „,fachund feldspezifischen Wissen“, dem „,berufspraktischen Wissen und Können“ und der ,professionellen Selbststeuerung“ als eines der wesentlichen Lernfelder für den Aufbau professioneller Handlungskompetenz im Kontext der Lehre in der Erwachsenenund Weiterbildung aus (vgl. Lencer und Strauch 2016, S. 7).

Obwohl sich die Erwachsenen- und Weiterbildung kontinuierlich ethisch positioniert ${ }^{1}$ und etwa zu ihrem gesellschaftlichen Auftrag umfassende Forschung und Theoriebildung betreibt, fehlt bislang ein bereichsspezifischer Forschungszusammenhang, in dem die moralischen Positionierungen der Profession mit dem Ziel reflektiert und diskutiert werden, eine begründete Berufsethik auszuarbeiten. Vor allem die konkreten Fragen der Lehr-Lerninteraktion, die für gewöhnlich im Rahmen von Berufsethiken behandelt werden, wurden bisher nicht bereichsethisch aufgearbeitet. Während eine Berufsethik eine Sammlung von normativen Aussagen ist, denen sich die Mitglieder einer Profession bzw. die Berufsträger, ähnlich wie in der ärztlichen Ethik, verpflichtet fühlen sollen, bildet eine Bereichsethik den jeweils dazugehörigen Forschungszusammenhang ab, der für einen bestimmten gesellschaftlichen Bereich, wie die Medizin oder die Pädagogik, versucht, ethische Fragen theoretisch zu erfassen und eine Berufsethik zu begründen und kritisch zu reflektieren. Im Unterschied zu klassischen Professionen gibt es in der Erwachsenenund Weiterbildung keine einheitliche Ausbildung und auch keine daran gekoppelte

\footnotetext{
1 Aktuell zeigt sich dies z.B. an der Kritik der „Nationalen Weiterbildungsstrategie“ (Bundesministerium für Arbeit und Soziales \& Bundesministerium für Bildung und Forschung 2019), welche z. B. in Blogeinträgen geäußert wird (z. B. vgl. Käpplinger 2019; Kilian 2019). Kritisiert wird, dass der Strategie eine zu enge Vorstellung von Weiterbildung zugrunde liege und diese vorrangig auf ökonomische Belange ausgerichtet sei. Stattdessen müsse es darum gehen, ,die Bedürfnisse der Menschen und der Weiterbildungslandschaft in ihrer Vielfalt öffentlich sichtbarer zu machen“ (Käpplinger 2019) und ,die Förderung einer Monokultur und von Partikularinteressen“ (ebd.) zu vermeiden. Anhand der Blogeinträge zeigt sich, wie die Erwachsenen- und Weiterbildung durch Berufung auf ihre Zuständigkeit bzw. ihr Mandat für das Lernen Erwachsener (vgl. Nittel 2000) Grenzüberschreitungen im Hinblick auf den eigenen funktionsspezifischen Verantwortungsbereich markiert und sich so ethisch positioniert.
} 
Lizensierung. Angesichts der Vielzahl von Unterrichtsfächern und Kontexte, in denen Erwachsenen- und Weiterbildung stattfindet, gibt es dafür auch gute Gründe. Allerdings führt das Fehlen einer Bereichsethik auch zu Problemen.

Für die Lehrenden in der Erwachsenenbildung hat dieses Defizit zur Folge, dass sie kaum Orientierung und Unterstützung erhalten, wenn sie in ihrem alläglichen Handeln mit moralischen Problemen konfrontiert werden und sich gefordert sehen, mit ethischen Fragen ${ }^{2}$ umgehen zu müssen, die z.B. den Umgang mit Vertraulichkeit, das Vermeiden von Interessenskonflikten oder den nicht-diskriminierenden Umgang mit Minoritäten betreffen (vgl. dazu Schrader und Spang 2019). Auch für die Professionalisierungsbemühungen in der Erwachsenen- und Weiterbildung ist die Vernachlässigung des Themas Berufsethik folgenreich. So sind der Professionalisierung der Erwachsenen- und Weiterbildung enge Grenzen gesetzt, wenn sich der Sektor nicht auf nationale und internationale berufsethische Standards verständigt (vgl. Martin und Langemeyer 2014, S. 56f.).

Vor diesem Hintergrund wird es immer wichtiger, dass die Erwachsenen- und Weiterbildung einen bereichsethischen Forschungszusammenhang entwickelt, in dem berufsethische Fragen von Lehrenden behandelt werden (Bernhardsson und Fuhr 2014, S. 4 ff.; S. 29 ff.; Arnold et al. 2017, S. $196 \mathrm{ff}$.). Wie diese dann in der Profession verankert werden, z. B. in der Ausbildung und Fortbildung, wäre eine weitere Frage, die hier aber nicht behandelt werden kann.

Das Anliegen des Beitrags besteht darin, Grundlagen für die Etablierung eines bereichsethischen Forschungsdiskurses zu ethischen Fragen von Lehrenden der Erwachsenen- und Weiterbildung zu schaffen. Dazu verfolge ich im Wesentlichen zwei Ziele: Zum einen wende ich mich in einem empirischen Zugang der Alltagsmoral von Lehrenden der Erwachsenen- und Weiterbildung zu. Dies ist m. E. erforderlich, da wir bisher nichts darüber wissen, in welchen Situationen moralische Probleme auftreten, mit welchen Themen diese einhergehen und mit welchen konkreten ethischen Fragen sich Lehrende konfrontiert sehen. Im Rahmen einer qualitativen Interviewstudie frage ich nach ethisch relevanten Handlungssituationen und konkreten ethischen Fragen, nach pädagogisch-ethischen Überzeugungen und ethischen Orientierungen, die sowohl bei der Entstehung ethischer Fragen als auch beim Umgang mit diesen eine zentrale Rolle spielen. Zudem besteht meine Absicht darin, auf Basis der empirischen Studie das Thema moralische Probleme und ethische Fragen von Lehrenden der Erwachsenen- und Weiterbildung als Forschungsfeld begrifflich zu erschließen. Auf Basis meiner Studienergebnisse stelle ich ein Modell vor, welches die wesentlichen Aspekte moralischer Probleme von Lehrenden der Erwachsenenund Weiterbildung beinhaltet. Anhand des Modells zeige ich auf, wie vielschichtig das Forschungsthema ist und welche Forschungsdesiderata sich ergeben.

\footnotetext{
2 In meinem Beitrag unterscheide ich nicht systematisch zwischen den Begriffen „Moral“ und „Ethik“. Ich verstehe darunter ein und denselben Sachverhalt, für den, je nachdem, worüber gerade gesprochen wird, unterschiedliche Begriffe benutzt werden. Während der Begriff „Ethik“ eher in theoretischen Kontexten gebraucht wird, in denen es um Reflexion geht und dabei zumeist von konkreten Einzelfällen abstrahiert wird, weist der Begriff „Moral“ eher einen konkreten Alltagsbezug auf. Von „Moral“ ist zumeist dann die Rede, wenn auf konkrete Situationen und Personen Bezug genommen wird. So spricht beispielsweise die Soziologie, welche sich dem alltäglichen Zusammenleben der Menschen zuwendet, von ,moralischer Kommunikation“, anstatt den Begriff „Ethik“ zu gebrauchen (vgl. dazu Bergmann und Luckmann 1999).
} 


\section{Forschungsstand und theoretische Grundlagen}

Im Folgenden skizziere ich zuerst den Stand der Entwicklung einer Berufsethik, dann den Forschungsstand zur Bereichsethik der Erwachsenen- und Weiterbildung. Bernhardsson und Fuhr (2014) haben vor wenigen Jahren den damaligen Stand der Entwicklung zu berufsethischen Standards in Verbänden der Erwachsenen- und Weiterbildung im deutschen Sprachraum erfasst. Sie argumentieren, dass insbesondere die Diversität des Feldes der Entwicklung eines Berufsverbandes, der möglichst alle Lehrenden im Feld erfolgreich organisieren würde, im Wege steht, weshalb die Erwachsenen- und Weiterbildung insgesamt noch weit von einer Berufsethik entfernt ist. Während sich nationale Träger, Organisationen und Verbände der allgemeinen Erwachsenenbildung bisher kaum dem Thema gewidmet haben, konnten Verbände, die Trainerinnen und Trainer in der betrieblichen Weiterbildung organisieren, erste Ansätze einer Berufsethik ausbilden. So haben das Forum Werteorientierung in der Weiterbildung e.V. (mit Unterstützung des Dachverbandes der Weiterbildungsorganisationen - DVWO) und der Berufsverband für Trainer, Berater und Coaches (BDVT) Berufskodizes entwickelt, die wichtige Bestimmungen wie etwa die Weiterbildungspflicht von Trainerinnen und Trainern enthalten. Das Forum Werteorientierung bewirbt die Berufsethik offensiv, vergibt ein Siegel und hat eine Beschwerdestelle sowie eine Beschwerdeordnung. Allerdings bleiben die Bestimmungen der Kodizes in weiten Teilen unspezifisch; es werden zwar Interpretationshilfen gegeben und der BDVT hat die Berufsethik in seine Ausbildungen integriert, aber kritische Diskussionen unter Einbezug der Verbandsmitglieder werden nicht angestoßen. Zudem wurden die berufsethischen Standards eher zentral, etwa durch speziell ins Leben gerufene Arbeitskreise entwickelt, ohne dass zuvor empirisch ermittelt wurde, mit welchen moralischen Problemen sich Lehrende und Trainerinnen und Trainer in ihrem Arbeitsalltag tatsächlich auseinandersetzen müssen. Es ist kein gesichertes Wissen darüber vorhanden, welche konkreten moralischen Fragen sich in Lehr-Lernkontexten der Erwachsenen- und Weiterbildung tatsächlich stellen, wie Berufspraktikerinnen und Berufspraktiker sie reflektieren und wie sie handelnd mit ihnen umgehen. Zudem ist nicht bekannt, welche Befürchtungen und Hoffnungen die verschiedenen Akteure mit Tendenzen verbinden, eine Berufsethik zu entwickeln.

Hinsichtlich der Bereichsethik weist die Erwachsenen- und Weiterbildung ähnliche Defizite auf wie bzgl. der Berufsethik (vgl. Bernhardsson-Laros 2016, S. 29). Vor allem aufgrund der Diversität ihres Feldes, hat ,die Erwachsenen- und Weiterbildung [...] bisher weder eine Berufsethik entwickelt, die in einem bekannten, allgemein akzeptierten und institutionalisierten Berufskodex ausgedrückt wäre, noch hat sie einen bereichsethischen Forschungszusammenhang ausgebildet" (vgl. Bernhardsson und Fuhr 2014, S. 41). Seitens der Sektion Erwachsenenbildung der Deutschen Gesellschaft für Erziehungswissenschaft gab es zwar entsprechende Bemühungen, bereichsethische Theoriebildungen anzustoßen (vgl. Gieseke et al. 1991; Hof 2010), diese reichten bisher jedoch nicht aus, um eine breite Diskussion sowie Forschungen zu einer erwachsenenpädagogischen Berufsethik anzuregen. Nach wie vor fehlen Untersuchungen, die sowohl nach den ,individuellen und gesellschaftlichen Bedingungen ethischer Herausforderungen in der erwachsenenpädagogischen Praxis“ (Schrader 2014, S. 25) als auch nach dem „Umgang [...] mit ihnen“ (ebd.) fragen. 
Stattdessen bleiben die ethischen Theoriebildungen mit der allgemeinen Theoriebildung zur Erwachsenen- und Weiterbildung verwoben (vgl. Fuhr 2011, S. 508 ff), was bedeutet, dass oftmals davon abgesehen wird, ethische Themen und Fragestellungen als solche zu benennen und zu reflektieren. Lediglich in der internationalen Debatte gibt es vereinzelt Untersuchungen zu ethischen Fragen (vgl. Gordon und Sork 2001; Brockett und Hiemstra 2004). Auf diese Lücke im erwachsenenpädagogischen Diskurs machen aktuell vor allem Einführungen in die Erwachsenen- und Weiterbildung aufmerksam (vgl. Fuhr 2011; Arnold et al. 2017; Schrader 2018). Sie weisen das Thema Ethik der Erwachsenen- und Weiterbildung als einen eigenständigen Inhaltsbereich aus und betonen den Forschungsbedarf zum Thema.

\section{Forschungsfragen}

Die Fragestellungen der im Folgenden vorgestellten Studie orientieren sich am typischen Aufbau berufsethischer Kodizes (vgl. Bernhardsson und Fuhr 2014, S. 46 ff). Traditionell sind Ethikkodizes inhaltlich in zwei Teile gegliedert und weisen zusätzlich einen Anwendungsbezug auf. Der erste Teil der Kodizes enthält Ideale, an denen sich die Profession ausrichten soll. Dies sind grundlegende Werte oder Prinzipien, wie z.B. die Würde des Menschen, das Streben nach Wahrheit, das Streben nach Exzellenz oder die Pflege demokratischer Prinzipien. In einem zweiten Teil werden die konkreten Standards, gegen die nicht verstoßen werden soll, festgelegt. Dies können Verpflichtungen gegenüber den Lernenden, den Auftraggebern oder der Profession sein. Im Idealfall weisen Ethikkodizes drittens einen Anwendungsbezug auf. Den Mitgliedern einer Profession ist bekannt, wie die Werte, Prinzipien und Standards interpretiert werden, wer in die Interpretationsprozesse einbezogen und wie mit Konflikten zwischen Bestimmungen und mit Interpretationsdifferenzen umgegangen werden soll. Ein weiterer Aspekt des Anwendungsbezugs ist, dass die niedergeschriebenen Prinzipien, Werte und Standards als Elemente für die ethische Entwicklung der jeweiligen Profession bzw. des jeweiligen Berufs genutzt werden. Kodifizierten Berufsethiken stehen bereichsethische Theoriebildungen und eine entsprechende Infrastruktur an Tagungen, Zeitschriften und Lehrbüchern zur Seite. Diese sollen dazu beitragen, die ethischen Prinzipien und Argumentationsweisen im jeweiligen Feld weiter zu entwickeln.

Gemäß diesen Vorannahmen über die idealtypische Anlage kodifizierter Berufsethiken habe ich insgesamt fünf Forschungsfragen bestimmt. Die ersten zwei Forschungsfragen $\mathrm{zu}$ ethisch relevanten Situationen und konkreten ethischen Fragen beziehen sich auf die Anwendungsbezüge (Teil 3 der Kodizes), die dritte Frage zu pädagogisch-ethischen Überzeugungen wurde mit Blick auf die konkreten ethischen Standards (Teil 2 der Kodizes) formuliert. Die vierte Forschungsfrage steht in Bezug zu den Idealen (Teil 1 der Kodizes). Und mit der fünften Frage soll darüber hinaus ermittelt werden, welche Hoffnungen und Befürchtungen Lehrende der Erwachsenen- und Weiterbildung in Hinblick auf eine mögliche Kodifizierung einer Berufsethik äußern. 
Ethisch relevante Situationen In welchen Situationen treten ethische Probleme bei Lehrenden auf?

Es geht darum zu ergründen, in welchen Anwendungsbezügen eine mögliche Berufsethik für Lehrende der Erwachsenen- und Weiterbildung stehen kann. Dazu soll ermittelt werden, in welchen beruflichen Situationen sich Lehrende gefordert sehen, ihr Handeln unter ethischen Gesichtspunkten zu reflektieren.

Ein Lehrender in der Weiterbildung für Pflegekräfte berichtet z.B. darüber, wie einzelne Teilnehmende eine Übung zur Anwendung neuer Beatmungsgeräte zu stören beginnen, indem sie die Geräte absichtlich falsch gebrauchen. Interessant an diesem Beispiel ist, dass der Lehrende die Situation für sich als ethisch-relevant identifiziert: Statt in den Störungen lediglich ein rein schulungsmethodisches Problem zu sehen, das empirisch daraufhin befragt werden müsse, wie Störungen am besten vermieden werden können, sieht er darin auch ein moralisches Problem.

Konkrete ethische Fragen Mit welchen konkreten ethischen Fragen und Problemen setzen sich Lehrende der Erwachsenen- und Weiterbildung auseinander?

Bei dieser Forschungsfrage geht es darum zu ergründen, welche konkreten ethischen Fragen aus den ethisch relevanten Situationen erwachsen.

Der Lehrende in unserem Beispiel berichtet darüber, dass er zumeist dazu tendiere, bei Störungen seine Überlegenheit zu demonstrieren, indem er diese, nach eigener Aussage, ,,üffisant“ übergeht, was einem rein schulungsmethodischen Umgang mit Störungen entspricht. In letzter Zeit würde er sich jedoch häufiger die Frage stellen, ob seine Art, Störungen zu übergehen, den Lernenden demonstriere, dass er sie nicht richtig ernst nehme, da er ihnen damit zeige, dass er an den Gründen für die Störungen gar nicht interessiert sei. Auf diese Weise beginnt der Lehrende den Umgang mit Störungen als eine ethische Frage zu reflektieren, bei der es um die Achtung den Störenden gegenüber geht. Für ihn stellt sich die konkrete ethische Frage, ob es generell wichtig ist, auf Störungen einzugehen, weil man dabei die Lernenden auch ernst nimmt, oder ob man sie auch übergehen kann und dann die Gefahr entsteht, dass man ihnen Achtung entzieht.

Pädagogisch-ethische Überzeugungen Welche pädagogisch-ethischen Überzeugungen sind für die Lehrenden handlungsleitend?

Ich frage nach den pädagogisch-ethischen Überzeugungen, an denen die Lehrenden ihr Handeln ausrichten. Die Überzeugungen drücken sich z. B. in Verpflichtungen gegenüber den Lernenden, den Auftraggebern, dem Arbeitgeber oder der Gesellschaft aus. Es wird untersucht, mithilfe welcher Überzeugungen Lehrende versuchen, ihre ethischen Fragen zu beantworten. Nur wenn situativ Konflikte zwischen unterschiedlichen Überzeugungen auftreten, entsteht ethischer Reflexionsbedarf.

Der Lehrende in unserem Beispiel ist sich unsicher, ob seine Praxis des Übergehens von Störungen durch das Demonstrieren von Überlegenheit, die seinem Selbstbild von sich als Lehrendem entspricht, mit den Anforderungen vereinbar ist, welche die Profession an den Umgang mit solchen Situationen stellt. Es konfligieren also zwei Überzeugungen, die sich aus Verpflichtungen gegenüber unterschiedlichen Akteuren ergeben. 
Ethische Orientierungen Woran orientieren sich Lehrende beim Umgang mit ethischen Fragen und Problemen?

Für die Frage nach den Idealen, an denen sich die Profession ausrichten soll, wird der Begriff „,ethische Orientierungen“ gewählt. Diese Terminologie soll dazu beitragen, eine Vielzahl möglicher Begründungsquellen (z. B. Prinzipien, Werte, implizite Modelle, Fachkulturen, Standards von Verbänden etc.), die Lehrende für die Bearbeitung ethischer Fragen nutzen, möglichst differenziert erfassen zu können.

Der Lehrende in unserem Beispiel ist vor einigen Jahren als Quereinsteiger zur Weiterbildung gekommen. Seitdem eignet er sich die für die Lehre nötigen pädagogischen Kompetenzen im Rahmen von Weiterbildungen an, wodurch, laut eigener Aussage, seine Identifikation mit der erwachsenenpädagogischen Fachkultur ständig zunehme. Seine ethische Orientierung an der Fachkultur bringt ihn nun dazu, seinen gewohnten Umgang mit Störungen ethisch zu hinterfragen.

Kodifizierte Berufsethik - Hoffnungen und Befürchtungen Welche Hoffnungen und Befürchtungen äußern Lehrende bzgl. einer kodifizierten Berufsethik?

Neben den möglichen Inhalten und den Anwendungsbezügen einer möglichen Berufsethik soll auch ergründet werden, wie Lehrende der Erwachsenen- und Weiterbildung insgesamt zu einer möglichen Kodifizierung einer Berufsethik stehen. Dazu wird danach gefragt, mit welchen Hoffnungen und Befürchtungen sie einer möglichen Kodifizierung entgegensehen.

Der Lehrende aus unserer Beispielsituation würde eine Berufsethik aufgrund ihrer Orientierungsfunktion für die Lehrenden begrüßen. Über weitere Vor- oder Nachteile habe er sich bisher keine Gedanken gemacht.

\section{Methodisches Vorgehen}

Es soll erfasst werden, in welchen Situationen Lehrende der Erwachsenen- und Weiterbildung ethische Fragen aufwerfen, mit welchen Überzeugungen sie diese konfrontieren und welche ethischen Orientierungen sie nutzen. Daher war es erforderlich Interviewpartnerinnen und -partner zu finden, die über möglichst viele solcher Probleme möglichst ausführlich berichten können. Die Auswahl der Interviewpartnerinnen und -partner erfolgte gemäß dem aus der Grounded Theory (Corbin und Strauss 2008) bekannten Prinzip des theoretischen Sampling (Strübing 2008):

- Mir bekannte Personen aus dem Feld der Erwachsenen- und Weiterbildung stellten Kontakt zu Personen her, von denen sie annahmen, dass sie viel zum Thema beitragen können.

- Weitere Gatekeeper waren Studierende des Masterstudiengangs Erwachsenenbildung der Pädagogischen Hochschule Freiburg. Diese stellten Kontakt zu relevanten Personen in ihren Praxisfeldern her, in denen sie im Rahmen des Studiums aktiv sind.

- Zudem stellten einzelne Interviewpartnerinnen und -partner Kontakt zu weiteren Lehrenden her. 
- Ehemalige Teilnehmende der Fortbildung „Kontaktstudium Erwachsenenbildung“ (Akademie für wissenschaftliche Weiterbildung Freiburg \& Pädagogische Hochschule Freiburg) wurden kontaktiert.

- Berufsverbände aus dem Trainerbereich, die bereits Ethikkodizes entwickelt haben, vermittelten den Kontakt zu Lehrenden, die an der Entwicklung des jeweiligen Ethikkodes beteiligt waren.

Auf Basis dieser Feldzugänge wurden insgesamt 19 leitfadengestützte Interviews mit Lehrenden aus unterschiedlichen Bereichen der Erwachsenen- und Weiterbildung geführt. 14 der 19 Interviews gingen in die Auswertung ein.

Soll Moral im Rahmen von Interviews thematisiert werden, ist laut Bergmann und Luckmann (vgl. 1999, S. 17 f.) zu beachten, dass es sich bei Moral um ein implizites Wissen handelt, das den Interviewten im Gespräch nicht direkt verfügbar ist. Denn Moral ,ist im wesentlichen Sinne gelebte Moral, die in den Handlungen und Entscheidungen, eben in [...] kommunikativen Akten existiert" (ebd., S. 18). Aufgrund dieser Vorannahme muss beachtet werden, dass Interviewpartnerinnen und -partner, die sich reflexiv den moralischen Aspekten ihrer Handlungen und Entscheidungen zuwenden, kommunikative Konstruktionsleistungen erbringen. Diese Konstruktionsleistungen können seitens der Interviewenden unterstützt werden, wenn sie ihre Interviewpartnerinnen und -partner zu Erzählungen und Beschreibungen anregen, bei denen Moral kommunikativ realisiert wird. Demgemäß konnten die Lehrenden in der vorliegenden Studie nicht aufgefordert werden, direkt über ethische Fragen und Probleme zu sprechen. Stattdessen wurden sie gebeten, über Situationen zu berichten, die sie dazu gebracht haben, ihr Handeln zu reflektieren. Gefragt wurde z.B. nach Situationen, in denen sie sich unsicher gefühlt haben oder in denen Konflikte aufkamen. In dieser frühen Phase der Interviews wurde darauf geachtet, dass Formulierungen gewählt werden, die ohne die Begriffe Ethik und Moral auskommen. Auf diese Weise sollte vermieden werden, dass die Interviewpartnerinnen und -partner vorschnell beginnen, im Hinblick auf die eigenen ethischen und moralischen Standpunkte zu argumentieren, was für eine kommunikative Realisierung ihrer Moral im Rahmen von Erzählungen und Beschreibungen hinderlich wäre. Entsprechend dieser methodologischen Vorannahmen wurde der Interviewleitfaden im fortschreitenden Forschungsprozess immer weiter durch Fragestrategien zu ethisch relevanten Situationen ergänzt. Als besonders ergiebig im Hinblick auf die Forschungsfragestellungen erwiesen sich die Fälle, bei denen die Interviewpartnerinnen und -partner über eine mehrjährige Berufserfahrung im Feld der Erwachsenen- und Weiterbildung verfügen. Dies ging zumeist auch mit einer größeren Sensibilität und Reflexionsfähigkeit in Bezug auf ethische Fragen einher.

Die Interviews wurden in einem zirkulären Prozess solange sukzessive durchgeführt und ausgewertet, bis sich erste Anzeichen einer theoretischen Sättigung (Strübing 2008) im Hinblick auf die Inhalte und die Struktur ethischer Fragen und Probleme einstellten. Die Datenauswertung erfolgte nach den Vorgaben der strukturierenden Inhaltsanalyse (Kuckartz 2012). Es handelt sich um ein Verfahren, bei dem theoretisch gewonnene Kategorien durch die Bildung von induktiven Subkategorien empirisch ausdifferenziert werden. In der vorliegenden Studie zeigte sich, dass 
auch die theoretisch angenommenen Hauptkategorien in diesem Prozess reformuliert werden mussten.

\section{Ergebnisse}

Im Zuge der Datenauswertung wurde ein Codesystem mit 620 Einträgen erstellt. Das Codesystem ist nach diesen fünf Oberthemen geordnet: (Abschn. 5.1), Ethischrelevante Situationen“ und „Ethische Fragen“, (Abschn. 5.2) „Pädagogisch-ethische Überzeugungen“, (Abschn. 5.3) „Ethische Orientierungen“, (Abschn. 5.4) „Berufsethik“ und (Abschn. 5.5) ,Entwicklung einer eigenen Ethik“. Die Oberthemen spiegeln, bis auf das Thema „Entwicklung einer eigenen Ethik“, die Forschungsfragen wider. Um einen Einblick in das Codesystem zu geben, stelle ich die fünf Oberthemen nacheinander anhand von Beispielen vor. Zudem führe ich etwas ausführlicher in zwei ethisch relevante Situationen ein. Die ausführlich beschriebenen Beispielsituationen nutze ich, um die fünf Oberhemen zu erläutern und zu zeigen, wie diese aufeinander bezogen sind.

\subsection{Ethisch relevante Situationen und ethische Fragen}

Die Daten bestätigen, dass sich konkrete ethische Fragen aus spezifischen Handlungssituationen ergeben, welche bei den Interviewten eine reflexive Auseinandersetzung mit den moralischen Aspekten ihrer beruflichen Tätigkeiten und Entscheidungen evozieren. Aus diesem Grund wurden ethisch relevante Situationen und ethische Fragen zusammen codiert (vgl. Tab. 1). Anhand des Obercodes „Interaktionskontexte" halte ich fest, ob die jeweiligen ethisch relevanten Situationen und die damit einhergehenden ethischen Fragen in der Interaktion mit Teilnehmenden oder in der Interaktion mit Trägern, Arbeitgebern oder Einrichtungsleitungen aufgetreten sind. Mit dem Obercode „Zeitpunkte“ wird danach unterschieden, ob die Probleme sich während der Auftragsakquise, der Auftragsdurchführung oder dem Auftragsabschluss ereigneten. Tab. 1 zeigt für jeden dieser drei Zeitpunkte ethisch relevante Situationen und ethische Fragen, die sich daraus für die Befragten ergeben können - dies jeweils für die Interaktion mit Teilnehmenden und für die Interaktion mit Trägern, Arbeitgebern, und Einrichtungsleitungen.

Es zeigt sich, dass Moral in den ethisch relevanten Situationen in zweierlei Hinsicht relevant wird. Lehrende identifizieren erstens Fragen der gegenseitigen Anerkennung und Achtung. Zweitens stellen sie Abgrenzungsfragen. Sie grenzen sich von als ,sach- und fachfremd“ erlebten Erwartungen ab, die von außen an sie herangetragen werden. Im Codesystem habe ich diese durchaus relevante Unterscheidung jedoch nicht berücksichtigt, da sich die ethisch relevanten Situationen nicht systematisch entlang der beiden Thematisierungsformen von Moral unterscheiden ließen. Bei der Beschäftigung mit Abgrenzungsfragen werden in der Regel auch Achtungsund Anerkennungsfragen mitverhandelt und vice versa (vgl. Krohn 1999, S. 6).

Die zwei folgenden Beispielsituationen geben einen tiefergehenden Einblick in den Zusammenhang von ethisch relevanten Situationen und ethischen Fragen. Wäh- 
Tab. 1 Übersicht ethisch relevante Situationen und ethische Fragen (Beispiele)

\begin{tabular}{|c|c|c|}
\hline \multirow[t]{2}{*}{ Zeitpunkte } & \multicolumn{2}{|l|}{ Interaktions-Kontexte } \\
\hline & Teilnehmende & $\begin{array}{l}\text { Träger, Arbeitgeber, Einrich- } \\
\text { tungsleitungen }\end{array}$ \\
\hline \multirow[t]{2}{*}{$\begin{array}{l}\text { Auftrags- } \\
\text { akquise }\end{array}$} & $\begin{array}{l}\text { Situation: Potentielle Teilnehmende fühlen sich vom } \\
\text { Thema angesprochen, wünschen sich aber andere Inhalte }\end{array}$ & $\begin{array}{l}\text { Situation: Inhalt eines sehr } \\
\text { lukrativen Auftrags sollen } \\
\text { manipulative Techniken sein }\end{array}$ \\
\hline & $\begin{array}{l}\text { Frage: Wie kann ich inhaltlichen Erwartungen einer- } \\
\text { seits wertschätzend begegnen, diesen andererseits aber } \\
\text { Grenzen setzen? }\end{array}$ & $\begin{array}{l}\text { Frage: Kann ich es mit mei- } \\
\text { nem Gewissen und meiner } \\
\text { gesellschaftlichen Verantwor- } \\
\text { tung vereinbaren, manipulative } \\
\text { Techniken zu vermitteln? }\end{array}$ \\
\hline \multirow[t]{2}{*}{$\begin{array}{l}\text { Auftrags- } \\
\text { durch- } \\
\text { führung }\end{array}$} & $\begin{array}{l}\text { Situation: Teilnehmende haben die Veranstaltung nur } \\
\text { gebucht, um einen schönen Ausflug zu haben }\end{array}$ & $\begin{array}{l}\text { Situation: Auftraggeber fordert } \\
\text { mich auf, Teilnehmende zu } \\
\text { kritisieren statt zu loben }\end{array}$ \\
\hline & $\begin{array}{l}\text { Frage: Kann ich Veranstaltungen vorzeitig beenden, } \\
\text { wenn die Teilnehmenden nicht die nötige Ernsthaftigkeit } \\
\text { für deren Durchführung mitbringen? }\end{array}$ & $\begin{array}{l}\text { Frage: Wie kann ich gegen- } \\
\text { über Auftraggebern meinen } \\
\text { anerkennenden u. wertschät- } \\
\text { zenden Seminarstil verteidi- } \\
\text { gen? }\end{array}$ \\
\hline \multirow[t]{2}{*}{$\begin{array}{l}\text { Auftrags- } \\
\text { abschluss }\end{array}$} & $\begin{array}{l}\text { Situation: Einzelne Teilnehmende haben die Veran- } \\
\text { staltung vorzeitig abgebrochen/haben den Kurs nicht } \\
\text { bestanden }\end{array}$ & $\begin{array}{l}\text { Situation: Der Auftraggeber } \\
\text { verlangt Feedback zu ein- } \\
\text { zelnen Teilnehmenden, ohne } \\
\text { dass die Teilnehmenden etwas } \\
\text { davon wissen sollen }\end{array}$ \\
\hline & $\begin{array}{l}\text { Frage: Ich bin mir häufig unsicher, ob es bei Nichtbeste- } \\
\text { hen oder bei Kursabbruch immer zwingend erforderlich } \\
\text { ist, das Gespräch mit den betreffenden Teilnehmenden } \\
\text { zu suchen, um mit ihnen z. B. über andere Weiterbil- } \\
\text { dungsmöglichkeiten zu sprechen. Wo beginnt und wo } \\
\text { endet meine Verantwortung für die Teilnehmenden? }\end{array}$ & $\begin{array}{l}\text { Frage: Ist die Bewertung von } \\
\text { Teilnehmenden, wenn dies } \\
\text { nicht expliziter Gegenstand der } \\
\text { Veranstaltung ist, mit meinen } \\
\text { Pflichten diesen gegenüber } \\
\text { vereinbar? }\end{array}$ \\
\hline
\end{tabular}

rend im ersten Beispiel vorrangig Achtungs- und Anerkennungsfragen behandelt werden, stehen im zweiten Beispiel Abgrenzungsfragen im Vordergrund.

\subsubsection{Beispiel 1: „Du hast 'n Nazi da drin“.}

Herr R. ist Kommunikationstrainer in der freien Wirtschaft. Im Verlauf der Durchführung eines mehrtätigen Kommunikationstrainings für ein Unternehmen teilte ihm der Vorgesetzte des Arbeitsbereichs, aus dem seine Kursteilnehmenden stammten, mit, dass ein Teilnehmer, den Herr R. bisher als sehr angenehm wahrgenommen hatte, ein überzeugter „Nazi“ sei. Im Interview reflektiert Herr R. darüber, dass in dieser Situation zwei Verpflichtungen in Konflikt zueinander geraten sind. Zum einen ist er gegenüber dem Teilnehmer zur Neutralität verpflichtet und könne diesen nicht aufgrund von Hörensagen diskriminierend behandeln. Zum anderen sieht er sich der Gesellschaft verpflichtet. Ein hoher moralischer Wert ist für ihn die Demokratie, und er möchte niemandem kommunikative Fähigkeiten vermitteln, von dem erwartet werden kann, dass er diese nutzt, um die Demokratie zu untergraben. Herr R. ist sich bewusst, dass er in einem moralischen Dilemma steckt: Er kann nicht beiden 
Verpflichtungen zugleich gerecht werden. Es stellt sich die Frage, inwieweit der überzeugte „Nazi“ Achtung und Anerkennung verdient.

\subsubsection{Beispiel 2: „Der meint mir sagen zu können welche Themen erlaubt sind und welche nicht"}

Herr S. ist Pastoralreferent, der im Rahmen seiner beruflichen Tätigkeit auch in der Erwachsenenbildung aktiv ist. Er berichtet darüber, dass er das Feld der Erwachsenenbildung für sich entdeckt hat und sich seit einigen Jahren immer stärker in diesem Bereich engagiert. Seine zunehmende Identifikation mit der Profession die Herr S. u. a. auf den Besuch berufsbegleitender Fortbildungen zur Erwachsenenund Weiterbildung zurückführt - geht laut seiner Schilderung mit einem zunehmenden Unbehagen bzgl. der Bedingungen der Durchführung von Erwachsenenbildung unter konfessioneller Trägerschaft einher. Ein Vertreter der Kirche versuche immer wieder, die Themen seiner Veranstaltungen mitzubestimmen, weil er die kirchliche Morallehre in den Veranstaltungen von Herrn S. nicht richtig vertreten sehe. Um sich gegenüber diesen - von ihm als fachfremd empfundenen - Eingriffen zu positionieren, verweist er auf seinen Bildungsauftrag, der auch darin bestehe, möglichst viele Menschen, auch mit eher liberalen Auffassungen, anzusprechen. Ein Problem für ihn ist jedoch, dass er konservativ eingestellten Gemeindemitgliedern keine Anlaufstelle mehr bietet. Herr S. sieht darin ein unauflösbares Dilemma konfessioneller Erwachsenenbildung, das ihn darüber nachdenken lässt, sich eine Anstellung als Erwachsenenbildner unter anderer Trägerschaft zu suchen. Für Herrn S. steht die Frage der Abgrenzung der Erwachsenenbildung von der Theologie im Vordergrund.

\subsection{Pädagogisch-ethische Überzeugungen}

Wie in den beiden Beispielen deutlich wird, spielen in den Erzählungen und Beschreibungen ethisch relevanter Handlungssituationen Konflikte bzw. Widersprüche zwischen pädagogisch-ethischen Überzeugungen eine wichtige Rolle. Während im ersten Beispiel (Kommunikationstrainer) die Verpflichtung gegenüber den Teilnehmenden und die Verpflichtung gegenüber der Gesellschaft in Konflikt zueinander geraten, konfligieren im zweiten Beispiel (Pastoralreferent) mehrere pädagogischethische Überzeugungen. In der Situation, sich gegen „fachfremde“ Eingriffe von außen wehren zu müssen, scheint sich der Pastoralreferent in einem Konfliktfeld zwischen den Verantwortlichkeiten gegenüber dem Arbeitgeber, den Lernenden, der Gesellschaft, der Profession sowie hinsichtlich des eigenen - sich neu entwickelnden - Selbstbildes als Erwachsenenpädagoge wiederzufinden.

In den Interviews wurden pädagogisch-ethische Überzeugungen im Hinblick auf die eigenen Verantwortlichkeiten und Pflichten, das Selbstbild, die Teilnehmenden und Lehrenden der Erwachsenen- und Weiterbildung genannt (siehe auch Tab. 2).

\subsection{Ethische Orientierungen}

Für die Entstehung und spezifische Bearbeitung von ethischen Fragen sind neben den pädagogisch-ethischen Überzeugungen auch die ethischen Orientierungen relevant. 
Tab. 2 Pädagogisch-ethische Überzeugungen (Beispiele)

\begin{tabular}{ll}
\hline Verantwortlichkeiten & Ich muss ethisch handeln und stehe in einem „Spannungsfeld“ \\
und Pflichten & Ich bin den Lernenden verpflichtet \\
& Ich bin dem Auftraggeber verpflichtet \\
& Ich bin der Gesellschaft verpflichtet \\
& Ich verstehe mich als Facilitator \\
Selbstbild & Ich bin ein politischer Mensch \\
& Ich stehe gerne vor Menschen (,Rampensau“) \\
& Es ist normal, dass nicht alle Teilnehmenden dich mögen \\
Teilnehmende & Teilnehmende stehen (immer häufiger) unter Zeitdruck \\
& Lernende suchen gezielt Dozierende (nicht WB-Einrichtung) \\
& Lehren muss man lernen \\
Lehrende & Lehrende und Lernende bilden eine Lerngemeinschaft \\
& Lehrende sind ,Ideenklauer“ \\
\hline
\end{tabular}

Beispiele für ethische Orientierungen, die im Codesystem festgehalten wurden, sind „Grundgesetz“, „Demokratie“, „Menschenrechte“, „mein geheimer Auftrag“ und „Fachkulturen“.

Im erstgenannten Beispiel des Kommunikationstrainers Herr R. zeigt sich, wie eine ethische Orientierung sowohl an der Entstehung als auch an der Bearbeitung einer ethischen Frage beteiligt ist. Die Demokratie stellt für Herrn R. einen hohen moralischen Wert dar. Als ihm mitgeteilt wird, dass ein „Nazi“ an seinem Kurs teilnimmt, hat er zunächst darüber nachgedacht, im Sinne seiner Orientierung an der Demokratie zu handeln. Er will keine kommunikativen Fähigkeiten an die ,falschen Leute“ vermitteln, die diese später missbrauchen könnten, um die Demokratie zu untergraben. Die Konsequenz wäre gewesen, den Teilnehmer anders als andere Teilnehmende zu behandeln oder ihn sogar aus dem Kurs auszuschließen. Herr R. stellt jedoch fest, dass dieser Handlungsentwurf gegen das Grundgesetz verstößt, welches für ihn eine andere wichtige ethische Orientierung ist, aus dem er seine Handlungsprinzipien ableitet. Er verweist diesbezüglich auf Artikel 3, gemäß dem niemand wegen seines Geschlechts, seiner Abstammung, seiner Rasse, seiner Sprache, seiner Heimat und Herkunft, seines Glaubens, seiner religiösen oder politischen Anschauungen benachteiligt oder bevorzugt werden darf. In der Situation schien es Herrn R. unmöglich, sowohl der pädagogisch-ethischen Überzeugung gerecht zu werden, sich für den Demokratieerhalt einzusetzen, als auch der pädagogisch-ethischen Überzeugung zu entsprechen, Kursteilnehmende nicht zu diskriminieren. Er bearbeitet das Dilemma, indem er den für ihn zunächst naheliegenden wertbezogenen Handlungsentwurf (Demokratieverteidigung) daraufhin überprüft, ob er auch mit universell gültigen Handlungsnormen (dem Grundgesetz) vereinbar ist. Abschließend stellt Herr R. fest, dass er mit seinem Ansatz zur Verteidigung der Demokratie gegen ein grundlegendes Prinzip der Demokratie verstoßen hätte.

Im zweiten Beispiel des Pastoralreferenten stellen sich mindestens zwei konkrete ethische Fragen. Zum einen behandelt Herr S. die Frage, wie er ,fachfremde“ Eingriffe in seinen Tätigkeitsbereich abwenden und sich gegenüber diesen positionieren kann. Zum anderen stellt sich für ihn die Frage, inwiefern er es rechtfertigen kann, dass sein liberaler und inklusiver Ansatz konservativ eingestellte Menschen - eine Kernklientel des Trägers - von einer Teilnahme an seinen Veranstaltungen abhält. 
Vor dem Hintergrund dieser Fragen scheint es ihm nur schwer möglich zu sein, seine pädagogisch-ethischen Überzeugungen - Verantwortlichkeiten gegenüber dem Arbeitgeber, den Lernenden, der Gesellschaft, der Profession, sowie hinsichtlich des eigenen Selbstbildes als Erwachsenenpädagoge - in Einklang miteinander zu bringen. Wesentlich relevant für die Bearbeitung der Fragen scheint für Herrn S. die ethische Orientierung „Fachkulturen“ zu sein. Er berichtet, dass es ihm einerseits mit zunehmender Orientierung an der erwachsenenpädagogischen Fachkultur immer besser gelingt, sich gegenüber den Eingriffsversuchen von außen zu behaupten, er dadurch aber andererseits verstärkt auf Unvereinbarkeiten zwischen seinem erwachsenenpädagogischen Handeln und der kirchlichen Morallehre stößt. Insgesamt betrachtet geben dieser und weitere Fälle aus dem Sample Hinweise darauf, dass sich aus einer zunehmenden Identifikation mit der erwachsenenpädagogischen Fachkultur und einem wachsenden professionellen Engagement eigens ethische Fragen ergeben können, mit denen sich die Lehrenden der Erwachsenen- und Weiterbildung auseinandersetzen müssen.

\subsection{Hoffnungen und Befürchtungen bezüglich einer kodifizierten Berufsethik}

An den Antworten der Interviewpartnerinnen und -partner auf die Frage, mit welchen Hoffnungen oder Befürchtungen sie einer Berufsethik entgegensehen würden, zeigt sich ein ambivalentes Bild. Generell finden sie das Thema Berufsethik wichtig und sind der Meinung, dass es sowohl im Fach als auch in der Profession stärker als bisher diskutiert werden sollte. Im Hinblick auf eine kodifizierte Berufsethik fallen die Antworten jedoch ambivalent aus. So äußerten sie beispielsweise, dass kein Bedarf für eine solche Ethik vorhanden sei (da bereits ein unausgesprochener Konsens zwischen den Lehrenden bestehe), dass eine kodifizierte Berufsethik dem kollegialen Austausch zugutekommen würde, dass eine Berufsethik nur einen symbolischen Charakter haben könne und dass eine kodifizierte Berufsethik auch die Auftraggeber bzw. Arbeitgeber in die Pflicht nehmen müsste. Insgesamt betrachtet zeigt sich, dass die Befragten die Orientierung in ethischen Fragen, die mit einer kodifizierten Berufsethik einhergehen würde, generell begrüßen, einer Festlegung auf eine einzelne Berufsethik jedoch eher kritisch gegenüberstehen.

\subsection{Entwicklung einer eigenen Ethik}

Bei dem Obercode „Entwicklung einer eigenen Ethik“ handelt es sich um ein Querschnittsthema, das ich in den Interviewdaten identifizieren konnte. Ähnlich wie beim Obercode ,ethische Orientierungen,, gibt es auch hier Hinweise dafür, dass ein Engagement bzgl. der erwachsenenpädagogischen Profession mit einer ethischen Sensibilität und Reflexionskompetenz einherzugehen scheint. Vor allem die Interviewten, die schon länger als zehn Jahre als Lehrende im Feld der Erwachsenen- und Weiterbildung tätig sind, beschreiben anhand von Entwicklungsthemen, wie sich ihre persönliche Berufsethik herausgebildet hat und wie sich ihre pädagogisch-ethischen Überzeugungen verändert und weiterentwickelt haben. So sind sie nun z. B. überzeugt davon, dass es besser ist, sich ethischen Problemen zu stellen als diesen aus dem Weg zu gehen; oder sie wissen nun sehr deutlich, welche Aufträge 
sie annehmen und welche nicht; oder es ist ihnen heute viel klarer als zu Beginn ihrer beruflichen Karriere, was für eine Lehrende bzw. ein Lehrender sie sein wollen. Dies scheint damit einherzugehen, dass sie nun eher bereit sind, zu eigenen Idealen, wie z. B. einem ,geheimen Auftrag“ (z. B. Themen gesellschaftskritisch behandeln), zu stehen. Zudem gibt das Querschnittsthema Hinweise darauf, dass Lehrende erst mit zunehmender Berufserfahrung beginnen, ihr Handeln als ethisch relevant zu betrachten und als solches zu reflektieren.

\section{Entwicklung eines Forschungsmodells zu moralischen Problemen}

Die Studie zeigt, dass ethisch relevante Situationen und ethische Fragen in allen Kontexten der Lehr-Lerninteraktion der Erwachsenen- und Weiterbildung auftreten können. Sie können sich während der Auftragsakquise, der Auftragsdurchführung und dem Auftragsabschluss jeweils in der Auseinandersetzung mit Lehrenden, Arbeitgebern und Trägern einstellen. Insgesamt betrachtet gibt die inhaltsanalytische Studie - und hier insbesondere die induktive Kategorienbildung - Hinweise darauf, dass zwischen ethisch-relevanten Situationen, ethischen Fragen, pädagogischethischen Überzeugungen und ethischen Orientierungen wechselseitige Zusammenhänge bestehen (Abb. 1).

In zweierlei Hinsicht deutet sich an, dass die vier Dimensionen des Modells miteinander zusammenhängen:

- Konflikte zwischen pädagogisch-ethischen Überzeugungen scheinen ausschlaggebend dafür zu sein, ob eine Handlungssituation als ethisch relevant erlebt wird und ob daraus später konkrete ethische Fragen abgeleitet werden können. Nur wenn es Konflikte zwischen pädagogisch-ethischen Überzeugungen gibt, scheint es auch ethische Fragen zu geben.

- In den Interviews hat sich gezeigt, dass die Befragten sich auf ethische Orientierungen beziehen, wenn sie ihre pädagogisch-ethischen Überzeugungen spezifizieren müssen. Demzufolge werden konkrete ethische Fragen anscheinend nicht nur aus Widersprüchen zwischen unterschiedlichen pädagogisch-ethischen Überzeu-

Abb. 1 Vier Dimensionen moralischer Probleme

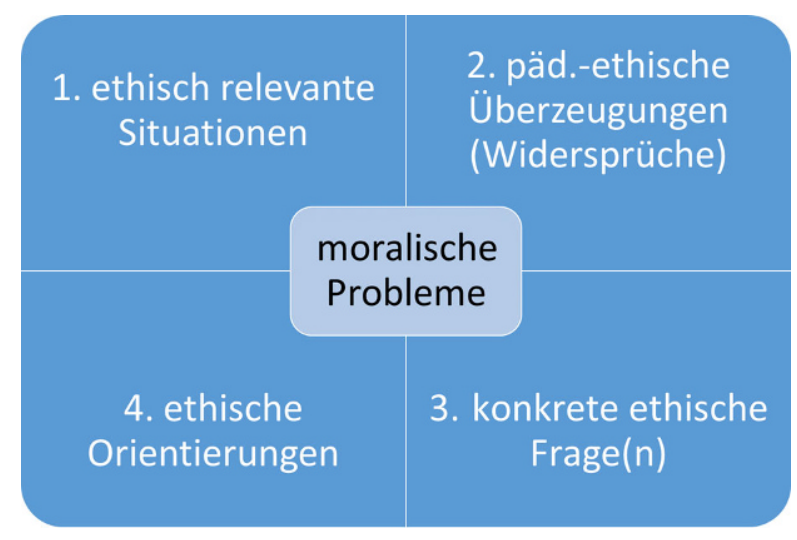


gungen abgeleitet, sondern sie werden, wie es scheint, erst gebildet, wenn auch ethische Orientierungen angelegt werden.

Gemäß dem skizzierten Modell setzen sich moralische Probleme aus vier Dimensionen zusammen. Folgen wir dem Modell, kann das erwachsenenpädagogische Handeln nicht begründet werden, wenn man sich auf eine dieser Dimensionen begrenzt. So ist es nicht ausreichend, dass Erwachsenenbildnerinnen und Erwachsenenbildner bloß pädagogisch-ethische Überzeugungen haben; sie müssen diese auch in konkrete ethische Fragen bringen können. Des Weiteren kann es problematisch sein, wenn eine Person pädagogisch-ethische Überzeugungen hat, sie aber gar nicht merkt, dass sie dagegen verstößt. Außerdem wäre es wichtig, dass sie sich daraufhin beobachtet, ob ihre pädagogisch-ethischen Überzeugungen und ihre ethischen Orientierungen zusammenpassen. Wie das oben genannte Beispiel zum Umgang mit dem vermeintlichen „Nazi“ zeigt, ist es erforderlich, dass die befragte Person merkt, dass es nicht zusammenpasst, einerseits für Menschenrechte $\mathrm{zu}$ sein und andererseits jemanden aus dem Lernprozess ausschließen zu wollen. Ein weiteres Problem könnte darin bestehen, dass eine Person ihren Umgang mit einer konkreten ethischen Situation ,,aus dem Bauch heraus“, ohne Rekurs auf eine pädagogischethische Überzeugung, begründet.

Vor diesem Hintergrund betrachtet, verstehe ich es als eine wesentliche Entwicklungsaufgabe für Erwachsenenpädagoginnen und -pädagogen, sich in allen vier Dimensionen moralischer Probleme weiter zu entwickeln, Zusammenhänge zwischen Situationen, Fragen, Überzeugungen und Orientierungen zu reflektieren, Konflikte zwischen Überzeugungen wahrzunehmen und zu bearbeiten, eine Sensibilität für moralische Probleme zu entwickeln, ihre ethischen Analysen mit allen relevanten Akteuren (andere Professionsmitglieder, Teilnehmerinnen und Teilnehmer, Träger, Auftraggeber, politische Akteure) zu kommunizieren und ihren Überzeugungen gemäß zu handeln. Vor allem wäre es zu kurz gegriffen, wenn Erwachsenenpädagoginnen und -pädagogen ausschließlich darauf bedacht wären, ihre Überzeugungen weiterzuentwickeln, ohne sie auf Situationen anzuwenden. Stattdessen ist es erforderlich, über alle vier Dimensionen moralischer Probleme Rechenschaft ablegen zu können und zwar in der Verbindung miteinander. Erst wenn diese Aufgabe erfüllt wird, kann von einer vollständigen moralischen Argumentation gesprochen werden.

Dies macht deutlich, in welche Richtung bereichsethische Forschung zu moralischen Problemen von Lehrenden der Erwachsenen- und Weiterbildung gehen sollte. Es darf nicht ausschließlich das Ziel sein, relevante pädagogisch-ethische Überzeugungen und ethische Orientierungen theoretisch zu reflektieren, wie dies bisher vornehmlich geschieht. Sondern es muss darum gehen, den skizierten Zusammenhang bzw. die Zusammenhänge zwischen den vier Dimensionen moralischer Probleme empirisch weiter zu erforschen. Nur auf diese Weise kann vermieden werden, dass Erwachsenenbildnerinnen und Erwachsenenbildner von Wissenschaftlerinnen und Wissenschaftlern und von Verbänden der Erwachsenenbildung mit Überzeugungen und Orientierungen konfrontiert werden, die sich nicht auf konkrete Situationen und die in ihnen vorhandenen Konflikte zwischen Überzeugungen beziehen und deswegen von den Handelnden nicht in konkretes Handeln in konkreten Situationen übersetzt werden können. Wenn moralische Probleme nicht in ihrer komplexen Struktur 
beforscht werden, entsteht die Gefahr, dass inkonsistente und unzusammenhängende (berufsethische) Erwartungen oder Anforderungen an erwachsenenpädagogische Lehrkräfte formuliert werden.

In Anbetracht des Modells, das ich als einen Versuch verstehe, die Komplexität moralischer Probleme im Kontext der Lehre der Erwachsenen- und Weiterbildung abzubilden, ist es erforderlich, gezielt einzelne Dimensionen und einzelne Zusammenhänge zwischen den Dimensionen zu beforschen. Ich beschränke mich im Folgenden auf zwei mögliche Forschungsperspektiven. Da meine Studie gezeigt hat, dass ethisch-relevante Situationen in allen Kontexten der Lehr-Lerninteraktion der Erwachsenen- und Weiterbildung auftreten können, wäre es bereichernd, wenn Studien durchgeführt würden, die ganz gezielt einen Kontext beforschen, beispielsweise ethisch relevante Situationen in der Auftragsakquise mit Arbeitgebern bzw. Trägern. Wünschenswert wäre es auch, zu erforschen, welche ethisch relevanten Situationen Lehrende mit wenig Berufserfahrung erleben. Wie Schwendemann (2018) gezeigt hat, kann es Lehrkräften an begrifflichen Instrumenten zu einer Reflexion konkreter moralischer Probleme fehlen. Auch ist es schwer, Widersprüche zwischen dem eigenen Denken und Handeln zu erkennen. Auch meine Erfahrungen mit der vorliegenden Studie bestätigen diese Herausforderung. Denn gerade Berufsanfängerinnen und -anfänger verfügen derzeit nicht notwendig über die begrifflichen Instrumente zu einer Reflexion konkreter moralischer Probleme. Zudem ,fehlt [es] offenkundig an Qualifizierungs- und Beratungsangeboten, die insbesondere unerfahrene und pädagogisch nicht vorgebildete Lehrkräfte darauf vorbereiten, moralische Konflikte zu erkennen und gegebenenfalls auch präventiv [...] zu bearbeiten“ (Schrader und Spang 2019, S. 54).

Eine Möglichkeit, sich dieser Herausforderung zu stellen, würde darin bestehen, die ethisch relevanten Situationen und ethischen Fragen, die ich im Rahmen meiner Studie ermitteln konnte, als Grundlage zu nutzen, um Fallbeispiele zu erarbeiten, die in Gruppendiskussionen diskutiert werden. Diese Gruppendiskussionen könnten sowohl wissenschaftlich ausgewertet werden, um moralische Probleme zu analysieren, als auch für Fortbildungen zur Entwicklung berufsethischer Reflexion (vgl. dazu Schwendemann 2018) eingesetzt werden.

Die Erwachsenenbildung sollte einen bereichsethischen Forschungsdiskurs ausbilden, der sich auf konkrete berufsethische Fragen bezieht und sich ethisch begründet positionieren, so wie dies auch andere Bereichsethiken tun. Wie das Beispiel des Pastoralreferenten nahelegt, der seine Erwachsenenbildungsaktivitäten vor zu viel theologischer Einflussnahme schützen will, könnten solche Forschungen und Positionierungen Lehrenden helfen, sich selbst berufsethisch zu reflektieren, sich mit anderen auszutauschen und sich ethisch zu positionieren. Ein Ansatz, der sowohl theoretisch als auch methodologisch geeignet wäre, um zu rekonstruieren, wie sich die Erwachsenen- und Weiterbildung etwa in Abgrenzung zu politischen oder ökonomischen Erwartungen - die z. B. durch Konzepte wie Lebenslanges Lernen oder Employability an sie herangetragen werden (vgl. dazu Bernhardsson-Laros 2018) ethisch positioniert, wäre z. B. die soziologische Konventionsanalyse (Alke 2019). Durch die Analyse der erwachsenenpädagogischen Diskurse im Hinblick auf die darin enthaltenen Konventionen könnten die ,spezifischen Wertigkeiten und Rationalitätsprinzipien“ (ebd., S. 465), mit denen sich die Erwachsenen- und Weiterbildung 
in Differenz zu anderen gesellschaftlichen Funktionsbereichen ethisch positioniert und stabilisiert, sichtbar gemacht werden.

\section{Schluss}

Im Rahmen einer qualitativen Interviewstudie wurde die theoretische Annahme weiter ausdifferenziert, dass sich moralische Probleme von Lehrenden der Erwachsenen- und Weiterbildung in ethisch relevanten Situationen, pädagogisch-ethischen Überzeugungen, konkreten ethischen Fragen und ethischen Orientierungen konstituieren, die in einem wechselseitigen Zusammenhang stehen. Die Studie gibt einen ersten Einblick, wie komplex moralische Probleme im Kontext der Lehre in der Erwachsenen- und Weiterbildung sind. Sie weist darauf hin, dass noch einige Forschungsbemühungen notwendig sind, um Grundlagen für eine forschungsbasierte Berufsethik für Lehrende der Erwachsenen- und Weiterbildung zu formulieren. Zudem wird deutlich, dass sich die befragten Lehrenden durchweg Unterstützung bzw. Orientierung beim Umgang mit moralischen Problemen wünschen und dass sie sich zu einer möglichen kodifizierten Berufsethik zumeist noch keine klare Meinung gebildet haben.

Vor diesem Hintergrund betrachtet wäre es wichtig, dass der erwachsenenpädagogische Diskurs sich in Richtung Berufs- und Bereichsethik entwickelt. Im Kontext der Professionalisierungsaktivitäten wurden im Rahmen des GRETA-Kompetenzmodells dafür bereits erste Weichen gestellt. Angesichts der Schwäche der Berufsverbände wäre es zu begrüßen, wenn das Thema Berufsethik im Rahmen dieser oder ähnlichen neu entstehenden Organisationsformen, die sich dem Aufbau erwachsenenpädagogischer Handlungskompetenzen verschreiben, institutionalisiert werden könnte. Auf diese Weise würde ein Ort für die Thematisierung und bereichsethische Erforschung berufsethischer Fragen geschaffen werden.

Open Access Dieser Artikel wird unter der Creative Commons Namensnennung 4.0 International Lizenz veröffentlicht, welche die Nutzung, Vervielfältigung, Bearbeitung, Verbreitung und Wiedergabe in jeglichem Medium und Format erlaubt, sofern Sie den/die ursprünglichen Autor(en) und die Quelle ordnungsgemäß nennen, einen Link zur Creative Commons Lizenz beifügen und angeben, ob Änderungen vorgenommen wurden.

Die in diesem Artikel enthaltenen Bilder und sonstiges Drittmaterial unterliegen ebenfalls der genannten Creative Commons Lizenz, sofern sich aus der Abbildungslegende nichts anderes ergibt. Sofern das betreffende Material nicht unter der genannten Creative Commons Lizenz steht und die betreffende Handlung nicht nach gesetzlichen Vorschriften erlaubt ist, ist für die oben aufgeführten Weiterverwendungen des Materials die Einwilligung des jeweiligen Rechteinhabers einzuholen.

Weitere Details zur Lizenz entnehmen Sie bitte der Lizenzinformation auf http://creativecommons.org/ licenses/by/4.0/deed.de.

\section{Literatur}

Alke, M. (2019). Rechtfertigungsstrategien in der Programmgestaltung in Volkshochschulen im Zuge veränderter Governance-Strukturen aus einer konventionentheoretischen Perspektive. In C. Imdorf, R. J. Leemann \& P. Gonon (Hrsg.), Bildung und Konventionen. Die „Economie des conventions “ in der Bildungsforschung (S. 461-482). Wiesbaden: Springer. 
Arnold, R., Nuissl, E., \& Rohs, M. (2017). Erwachsenenbildung. Eine Einführung in Grundlagen, Probleme Perspektiven. Baltmannsweiler: Schneider Hohengehren.

Bergmann, J., \& Luckmann, Th (1999). Moral und Kommunikation. In J. Bergmann \& T. Luckmann (Hrsg.), Struktur und Dynamik der Formen moralischer Kommunikation. Kommunikative Konstruktion von Moral, (Bd. 1, S. 13-38). Opladen: Westdeutscher Verlag.

Bernhardsson, N., \& Fuhr, Th (2014). Standards ethischen Handelns in Verbänden der Erwachsenenbildung. Zum Stand der Entwicklung einer erwachsenenpädagogischen Bereichsethik. Report. Zeitschrift für Weiterbildungsforschung. https://doi.org/10.3278/REP1401W

Bernhardsson-Laros, N. (2016). Die Leerstelle schließen. Probleme und Perspektiven einer Berufsethik für Erwachsenenbildung. DIE - Zeitschrift für Erwachsenenbildung. https://doi.org/10.3278/DIE1602W

Bernhardsson-Laros, N. (2018). Wertehorizont Beschäftigungsfähigkeit im Betrieb. Eine pädagogische Rekonstruktion. Wiesbaden: Springer.

Brockett, R. G., \& Hiemstra, R. (2004). Toward ethical practice. Malabar: SAGE.

Bundesministerium für Arbeit und Soziales, \& Bundesministerium für Bildung und Forschung (2019). Wissen teilen. Zukunft gestalten. Zusammen wachsen. Nationale Weiterbildungsstrategie. https:// www.bmas.de/SharedDocs/Downloads/DE/Thema-Aus-Weiterbildung/strategiepapier-nationaleweiterbildungsstrate-gie.pdf; jsessionid=986FA52FB44A4507F9CC0FDBBD283998?_blob= publicationFile\&v=6. Zugegriffen: 4. Dez. 2019.

Corbin, J. M., \& Strauss, A.L. (2008). Basics of qualitative research: Techniques and procedures for developing grounded theory (3. Aufl.). Los Angeles and California: SAGE.

Fuhr, Th (2011). Ethik der Erwachsenenbildung/Weiterbildung. In T. Fuhr, P. Gonon \& C. Hof (Hrsg.), Erwachsenenbildung/Weiterbildung. Handbuch Erziehungswissenschaft, (Bd. 4, S. 505-518). Paderborn: Schöningh.

Gieseke, W., Nuissl, E., \& Meueler, E. (Hrsg.). (1991). Ethische Prinzipien der Erwachsenenbildung: Verantwortlich für was und vor wem? Kassel: Gesamthochschule Kassel.

Gordon, W., \& Sork, T. J. (2001). Ethical issues and codes of ethics: Views of adult education practitioners in Canada and the United States. Adult Education Quarterly, 3, 202-218.

Hof, Ch (Hrsg.). (2010). Professionalität zwischen Praxis, Politik und Disziplin. Baltmannsweiler: Schneider Hohengehren.

Käpplinger, B. (2019). Ist das eine Strategie? Gastbeitrag im Blog von Jan Martin-Wiarda. https://www. jmwiarda.de/2019/06/13/ist-das-eine-strategie/. Zugegriffen: 4. Dez. 2019.

Kilian, L. (2019). Darf es ein bisschen mehr sein. Beitrag im wb-web. https://wb-web.de/aktuelles/darfes-ein-bisschen-mehr-erwachsenenbildung-sein.html. Zugegriffen: 4. Dez. 2019.

Krohn, W. (1999). Funktionen der Moralkommunikation. Soziale Systeme, 5(2), 313-338.

Kuckartz, U. (2012). Qualitative Inhaltsanalyse. Methoden, Praxis, Computerunterstützung (1. Aufl.). Wiesbaden: Springer VS.

Lencer, S., \& Strauch, A. (2016). Das GRETA-Kompetenzmodell für Lehrende in der Erwachsenen und Weiterbildung. https://www.die-bonn.de/doks/2016-erwachsenenbildung-02.pdf. Zugegriffen: 4. Dez. 2019.

Luhmann, N. (1993). Die Moral des Risikos und das Risiko der Moral. In G. Bechmann (Hrsg.), Risiko und Gesellschaft (S. 327-338). Opladen: Westdeutscher Verlag.

Martin, A., \& Langemeyer, I. (2014). Demografie, sozioökonomischer Status und Stand der Professionalisierung - das Personal in der Weiterbildung im Vergleich. In Deutsches Institut für Erwachsenenbildung (Hrsg.), Trends der Weiterbildung. DIE-Trendanalyse 2014. Bielefeld: W. Bertelsmann.

Nittel, D. (2000). Von der Mission zur Profession? Stand und Perspektiven der Verberuflichung in der Erwachsenenbildung. Bielefeld: W. Bertelsmann.

Schrader, J. (2014). Im Fokus. Ethik erwachsenenpädagogischen Handelns - alltäglich gefordert, selten bedacht. Report. Zeitschrift für Weiterbildungsforschung. https://doi.org/10.3278/REP1401W

Schrader, J. (2018). Lehren und Lernen in der Erwachsenen- und Weiterbildung. Bielefeld: W. Bertelsmann.

Schrader, J., \& Spang, F. (2019). Zum Umgang mit konfligierenden Werten in Integrationskursen. weiter bilden. DIE Zeitschrift für Erwachsenenbildung. https://doi.org/10.3278/WBDIE1904W

Schwendemann, N. (2018). Werthaltungen von Lehrkräften in der Erwachsenenbildung. Eine rekonstruktive Studie. Wiesbaden: Springer VS.

Strübing, J. (2008). Grounded Theory: Zur sozialtheoretischen und epistemologischen Fundierung des Verfahrend der empirisch begründeten Theoriebildung (2. Aufl.). Wiesbaden: Springer VS.

Publisher's Note Springer Nature remains neutral with regard to jurisdictional claims in published maps and institutional affiliations. 